\title{
A Scaling Law for the Snapback in Superconducting Accelerator Magnets
}

\author{
G. Ambrosio, P. Bauer, L. Bottura, M. Haverkamp, T. Pieloni, S. Sanfilippo, and G. Velev
}

\begin{abstract}
The decay of the sextupole component in the bending dipoles during injection and the subsequent snapback at the start of beam acceleration are issues of common concern for all superconducting colliders built or in construction. Recent studies performed on LHC and Tevatron dipole magnets revealed many similarities in the snapback characteristics. Some are expected, e.g. the effect of operational history. One particular similarity, however, is striking and is the subject of this paper. It appears that there is a simple linear relation between the amount of sextupole drift during the decay and the magnet current (or field) change during the ramp required to resolve the snapback. It is surprising that the linear correlation between snapback amplitude and snapback field holds very well for all magnets of the same family (e.g. Tevatron or LHC dipoles). In this paper we present the data collected to date and discuss a simple theory that explains the scaling found.
\end{abstract}

Index Terms-Current distribution, decay and snapback, magnetization reversal, superconducting accelerator magnets.

\section{INTRODUCTION}

$\mathbf{T}$ HE magnetic field in superconducting accelerators drifts during injection, as was found for the first time in the Tevatron collider [1]. This drift has a magnitude of a few units, i.e. $10^{-4}$ relative to the main field, and at both the Tevatron [2] and HERA colliders [3] requires active compensation to avoid beam instability, particle losses, or, simply, decreased performance because of emittance growth. The integrated drift in the accelerator is dominated by the contribution of the bending dipoles, and has been found to be systematic in all allowed harmonics of the dipole field (dipole $b_{1}$, sextupole $b_{3}$, decapole $b_{5} \ldots$ ). The present understanding attributes this drift to the decrease of the magnetization of the superconducting filaments induced by the local field changes that are associated with current redistribution in the Rutherford cables [4], [5]. Indeed, the interaction between current distribution and magnetization change could be demonstrated both through experiments [6] and simulation [7], and was found to be associated with a rotating magnetization component that averages out to a net decrease of the magnetic moment associated with the persistent currents in the filament, hence the name decay for this drift.

The characteristic time of current distribution is long, typically hundreds of $\mathrm{s}$ and more, and the decay is accordingly slow. For this reason, it is in principle possible to measure the decay of the low order harmonics directly on the beam and correct for

Manuscript received October 3, 2004.

G. Ambrosio, P. Bauer, and G. Velev are with FNAL, Batavia, IL, USA.

L. Bottura, T. Pieloni and S. Sanfilippo are with CERN, Geneva, Switzerland (e-mail: Luca.Bottura@ cern.ch).

M. Haverkamp was with Twente University, The Netherlands, and CERN, Geneva, Switzerland and is now with Metrolab.

Digital Object Identifier 10.1109/TASC.2005.849535

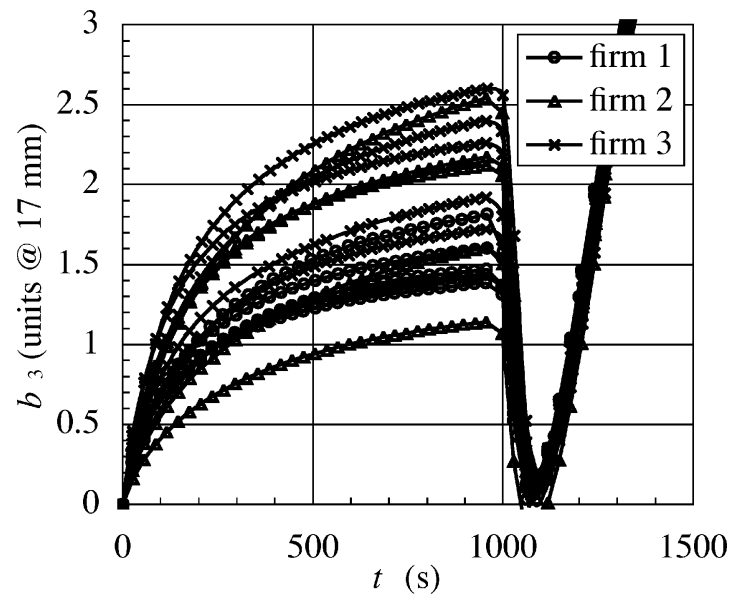

Fig. 1. Measured normal sextupole $b_{3}$ decay and snapback in few selected LHC dipoles from the three firms. The simulated particle injection plateau lasts $1000 \mathrm{~s}$. The snapback is the fast recovery of the value at the beginning of injection, followed by the increase of $b_{3}$ along the magnet load-line. All values have been arbitrarily shifted so that the initial value is zero.

it. However, at the end of injection, when the beam acceleration starts, the rotating magnetization component is wiped out by the change of the background field, and the magnetization state is brought back to a state close to that at the beginning of injection. This snapback happens within few $\mathrm{mT}$ of field change and can be fast, e.g. few s, depending on the ramp-rate of the magnets. Fig. 1 shows a typical example of decay and snapback measured in LHC dipoles during a simulated $1000 \mathrm{~s}$ injection and the acceleration ramp.

Because of the speed at which it happens, snapback is difficult to compensate directly from beam measurements. In addition, because it results from a change of a complex magnetization state, it is difficult to predict from simulations. A common practice for correction is hence to rely on measurements of on-line reference magnets to establish suitable correction waveforms [2]. In addition to the difficulties above, the magnitude of the decay and of the snapback vary considerably from magnet to magnet, and, for the same magnet, both depend strongly on the magnet powering history, and in particular the current cycle during the previous accelerator physics run. The selected results reported in Fig. 1 show a typical example of the variations observed among few of the magnets tested in the framework of the LHC series cold tests.

This apparent maze is becoming clearer in the light of the results presented in this paper. We started in early 2003 a collaborative effort between CERN and Fermilab to measure detailed snapback waveforms in LHC and Tevatron dipoles and we have found an inherent regularity that could both shed some light on 
TABLE I

PARAMETERS OF THE MEASUREMENT CyCle AND RANGE TESTED

\begin{tabular}{lcccc}
\hline Parameter & \multicolumn{2}{c}{ LHC dipoles } & \multicolumn{2}{c}{ Tevatron dipoles } \\
& nominal & range & nominal & range \\
\hline $\mathrm{I}_{\mathrm{FT}}(\mathrm{A})$ & 11850 & $4000-11850$ & 4333 & $2000-4333$ \\
$\mathrm{t}_{\mathrm{FT}}(\mathrm{min})$ & 30 & $5-60$ & 20 & $1-60$ \\
$\mathrm{I}_{\mathrm{FB}}(\mathrm{A})$ & 350 & - & 666 & - \\
$\mathrm{t}_{\mathrm{FB}}(\mathrm{min})$ & 0 & & 1 & $1-30$ \\
$\mathrm{I}_{\text {inj }}(\mathrm{A})$ & 760 & $550-1050$ & 666 & - \\
$\mathrm{t}_{\text {inj }}(\mathrm{min})$ & 17 & $17-170$ & 30 & $5-30$ \\
\hline
\end{tabular}

the physics and make the forecast of the field change during snapback easier. We present here the measurement results, the analysis procedure and the empirical scaling we found, together with a simple theory that explains the scaling.

\section{EXPERIMENTAL}

\section{A. Magnets Measured and Test Procedure}

The magnets tested are dipoles of the prototype and series production for the $\mathrm{LHC}$ (prototype $\mathrm{MBP} 2 \mathrm{O} 2$ and series magnets MB1001, MB1009, MB1024, MB2020, MB3005), and spare Tevatron dipoles (TB0269, TB0834, TC1052, TC1220). Magnets of the same family have the same construction features, but the two families have obviously great differences in the coil and iron yoke design. Details on the two magnet families can be found in [8] and [9]. The measurement procedure for the data discussed here was the following:

- quench to erase the memory of previous current cycles;

- ramp-up to a flat-top current $\mathrm{I}_{\mathrm{FT}}$ for a time $\mathrm{t}_{\mathrm{FT}}$;

- ramp-down to a flat-bottom $\mathrm{I}_{\mathrm{FB}}$ for a time $\mathrm{t}_{\mathrm{FB}}$;

- (magnetization reset at $400 \mathrm{~A}$ for the Tevatron dipoles);

- ramp-up to injection current $I_{i n j}$ for a time $t_{i n j}$;

- measurement of the snapback during the ramp.

Table I contains the nominal values of the current and times that define the measurement cycles for the two magnet families, as well as the typical range of variations tested. Various combinations of parametric variations were tested on different magnets, for a total of 18 test runs on LHC magnets and 43 test runs on the Tevatron magnets.

\section{B. Measurement Method}

As discussed in the introduction, snapback is a relatively fast phenomenon, that can take within few seconds. An instrument with a suitable resolution in time is needed in order to measure its evolution accurately. To this aim we have developed probes that use Hall plates as field sensors, arranged geometrically so to yield a signal proportional to the sextupole component in a dipole magnet. Two similar probes of this type were used to test the LHC and Tevatron dipoles, achieving a measurement rate of $10 \mathrm{~Hz}$ with a resolution of $b_{3}$ at the level of 0.02 units, well suited for the characterization of the snapback. Details on the probes construction and performance can be found in [10]-[12]

\section{MEASUREMENT Results AND SCALing LaW}

In the analysis of the measurements we have focused on the evolution of the normal sextupole $b_{3}$ as a function of current

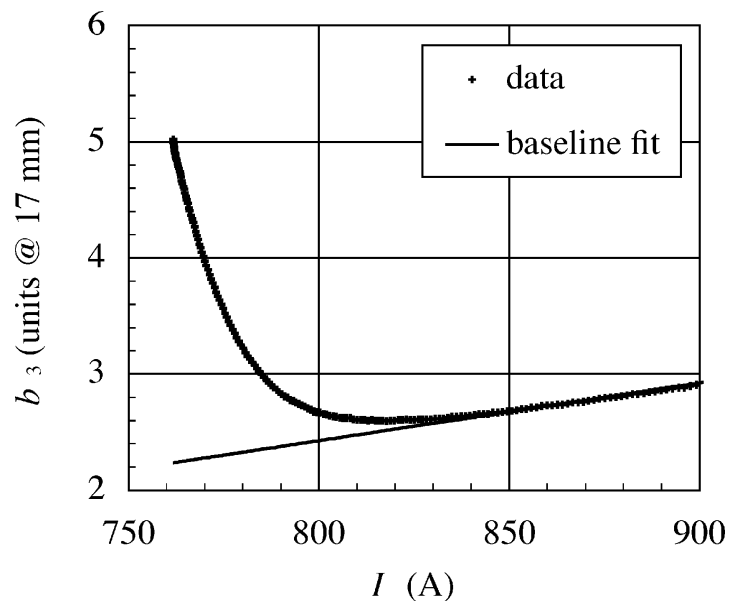

Fig. 2. Sextupole plotted as a function of current during snapback and the initial ramp. The baseline fit, extrapolated down to injection current, is also shown. Measurement on LHC dipole MB3005, following a standard pre-cycle and an injection lasting $2000 \mathrm{~s}$.

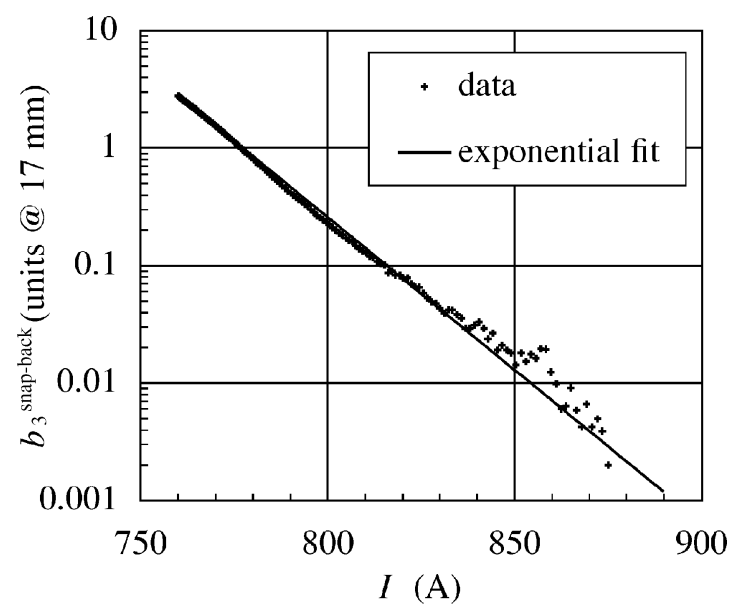

Fig. 3. Sextupole change with respect to the baseline ramp, as obtained from the data in Fig. 2, plotted in semi-log scale. The exponential fit (2) is reported for comparison. The fit parameters are $\Delta b_{3}=2.81$ units and $\Delta I=16.7 \mathrm{~A}$.

during the snapback and the following ramp. To compare different measurements we have computed the change in sextupole $b_{3}^{\text {snapback }}$ with respect to a baseline ramp $b_{3}^{\text {baseline }}$, i.e. as would be measured with no stop at injection. The latter was estimated directly from the measurements of each cycle, fitting the data after the end of the snapback with a parabola and extrapolating the fit down to the injection current.

In Fig. 2 we report an example of the measured $b_{3}$ (magnet MB3005, standard pre-cycle, 2000 s injection) together with the fitted parabola for the ramp-up baseline ramp $b_{3}^{\text {baseline extrapo- }}$ lated to injection field. The sextupole change is obtained as:

$$
b_{3}^{\text {snapback }}=b_{3}-b_{3}^{\text {baseline }} .
$$

In the current range considered, the fit of $b_{3}^{\text {baseline }}$ deviates only slightly from a straight line and the extrapolation of the baseline ramp is expected to introduce negligible errors in the evaluation of $b_{3}^{\text {snapback }}$.

The change in sextupole during the snapback, $b_{3}^{\text {snapback }}$, as a function of current, is reported in Fig. 3 for the same measurement plotted in Fig. 2. We see in the semi-log plot used there 


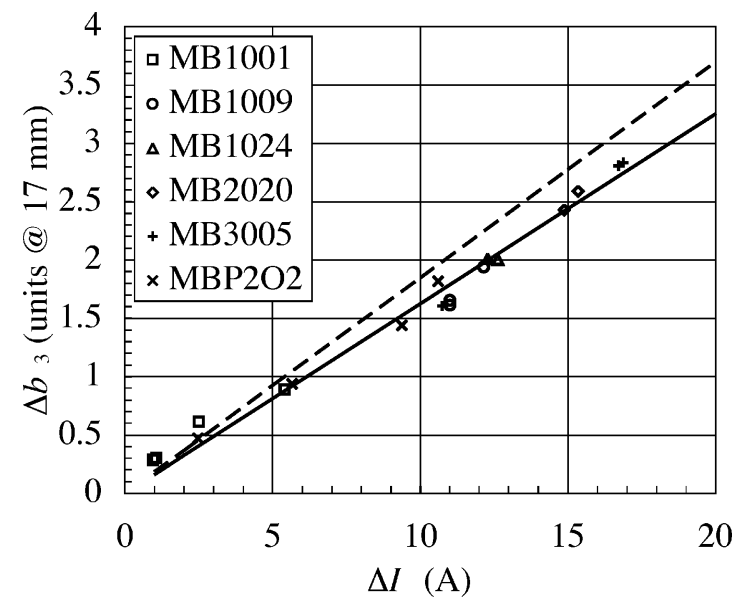

Fig. 4. Scatter plot of the fit parameters $\Delta b_{3}$ and $\Delta I$ as obtained from the analysis of the measurements performed on the LHC dipoles. Multiple symbols correspond to different powering cycles performed on the same magnet (see Table I). The solid line is the best fit to the data, the dashed line is the result of the theory discussed in Section IV.

that the function $b_{3}^{\text {snapback }}(I)$ can be approximated well by an exponential of the following form:

$$
b_{3}^{\text {snapback }}(I)=\Delta b_{3} e^{-\frac{I-I_{i n j}}{\Delta I}}
$$

where $I$ is the instantaneous value of the current, initially at the injection value $I_{i n j}$. The two fitting constants are the snapback amplitude $\Delta b_{3}$ and the current change $\Delta I$.

As discussed in the introduction, the sextupole snapback has a large variation among magnets, and is correlated with the powering history. This variation results in different fitting constants $\Delta b_{3}$ and $\Delta I$ for each of the different experiments performed. In general long flat-top at high current yields a deeper snapback, i.e. a larger $\Delta b_{3}$, while waiting before injection (e.g. at flat-bottom) tends to reduce the phenomenon, i.e. a smaller value of $\Delta b_{3}$. A very interesting observation is that the current change $\Delta I$ characterizing the length of the snapback is also a function of the powering history, and that in general a large sextupole change $\Delta b_{3}$ is associated with large current change to reach the baseline ramp. In other words, the fitting parameters $\Delta b_{3}$ and $\Delta I$ in the exponential (2) appear to be strongly correlated.

This result was first found during tests of the LHC magnets [7], and is clearly visible in the scatter plot of Figs. 4 and 5, reporting the fitting parameters obtained for all LHC and Tevatron magnets tested. The correlation can be written explicitly:

$$
\Delta b_{3}=\beta_{3} \Delta I
$$

where the coefficient $\beta_{3}$ has values of 0.16 and 0.22 units/A for the LHC and Tevatron data respectively. A similar correlation was also found for the decapole snapback [13], although on the number of measurements available is smaller.

As clear from Figs. 4 and 5, (3) is a scaling law that appears to hold with good approximation for all magnets of the same family, as well as for any powering cycle preceding the injection and acceleration. In short, it gives the possibility to forecast the snapback waveform using (2) once only one of the two fitting

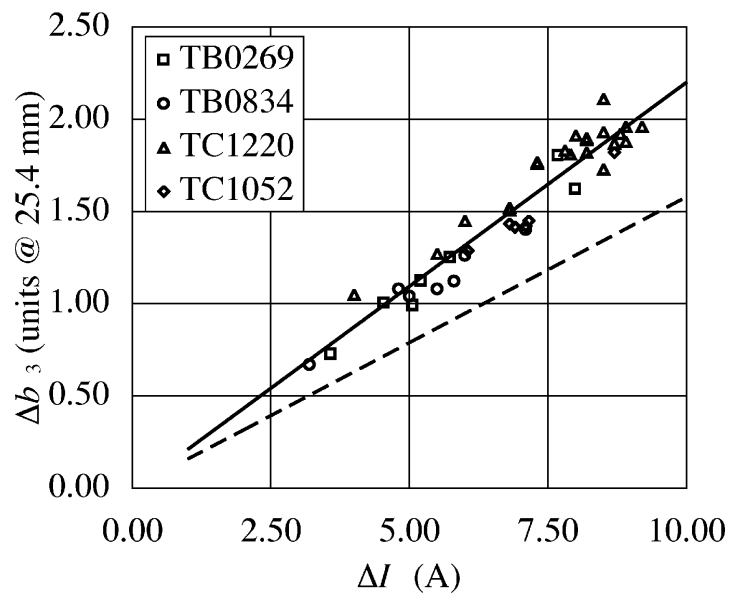

Fig. 5. Scatter plot of the fit parameters $\Delta b_{3}$ and $\Delta I$ as obtained from the analysis of the measurements performed on the Tevatron dipoles. Same conventions on symbols and lines as in Fig. 4.

parameters $\Delta b_{3}$ or $\Delta_{I}$ is known. The second parameter can be determined using (3). We have proposed elsewhere a method to exploit this finding for the control of superconducting accelerators [13].

What is important in this context is that the correlation hints to an inherent regularity in the behavior of all magnets in the same family. This happens in spite of the variation of parameters such as inter-strand resistance which are expected to be the main driver for the decay and the origin of the ensuing snapback. In the next section we discuss a possible reason for this regularity.

\section{A Simple THEORY FOR SNAPBACK}

An interpretation of the results described above is provided by the findings reported in [7]. As discussed there, when a strand is initially magnetized in a background field $B_{0}$, and then subjected to a rotating field change, the local distribution of shielding currents becomes very complex and the average magnetic moment in the direction of $B_{0}$ is reduced. Once the background field is increased again, however, the change in magnetization in the direction parallel to the background field can be fitted very well with a simple exponential expression:

$$
M(B)=\Delta M e^{-\frac{B-B_{0}}{\Delta B}}
$$

In the expression above $\Delta M$ and $\Delta B$ are fitting constants that follow the experimental correlation:

$$
\mu_{0} \Delta M=A \Delta B
$$

where $A$ is a nondimensional constant equal to $0.46 \pm 0.04$ for the strand used in the inner cable of the LHC dipoles [7].

During snapback, each strand in the magnet coil undergoes a magnetization change as given by (4), where the initial magnetization state, and thus $\Delta M$, is unknown, but the relation (5) holds. We can imagine at this point to compute the sextupole field change during snapback adding the contribution of the magnetization change from each strand:

$$
b_{3}^{\text {snapback }}(B)=\sum_{i} \chi_{3, i} \Delta M_{i} e^{-\frac{B_{i}-B_{i, \text { injection }}}{\Delta B_{i}}}
$$


where the sum is extended over all strands in a coil and $\chi_{3, \mathrm{i}}$ is the coefficient of proportionality between a magnetic moment located at strand $i$ and the contribution to the sextupole field. This is a geometric constant that can be computed a priori for a given magnet using standard field theory [14]. Making now use of (5), and substituting for the local field the product of the local transfer function $T_{i}$ and the current $I$, (6) can be written as follows:

$$
b_{3}^{\text {snapback }}(I)=\sum_{i}\left(\chi_{3, i} \frac{A}{\mu_{0}} T_{i} \Delta I_{i} e^{-\frac{I-I_{\text {injection }}}{\Delta I_{i}}}\right)
$$

As we seek here only a broad confirmation of the order of magnitude of the scaling, we approximate the local transfer function and current increments in the sum using the average values over the whole cross section, i.e.:

$$
b_{3}^{\text {snapback }}(I) \approx \frac{A}{\mu_{0}} T\left(\sum_{i} \chi_{3, i}\right) \Delta I e^{-\frac{I-I_{\text {injection }}}{\Delta I}}
$$

We note that (8) explains the exponential dependency (2) found experimentally. Moreover, comparing terms in (8) vs. (2) and (3), we obtain an analytical expression for the scaling coefficient $\beta_{3}$ :

$$
\beta_{3} \approx \frac{A}{\mu_{0}} T\left(\sum_{i} \chi_{3, i}\right)
$$

Eq. (9) was evaluated numerically for the two dipole designs considered. The values of the average transfer function $T$ are 0.39 and $0.61 \mathrm{mT} / \mathrm{A}$ respectively for the LHC and Tevatron dipoles. The corresponding values of $\beta_{3}$ calculated from (9) are 0.19 and 0.16 units/A for LHC and Tevatron. In Figs. 4 and 5 we compare the scaling obtained analytically (dashed line) to the linear fit of the experimental results (solid line). Although the theory described here is simple, it provides a good fit of the measured behavior, which is a very satisfactory result.

\section{CONCLUSION}

We have derived what we consider a universal scaling law for the snapback of allowed harmonics, and in particular the sextupole, in accelerator dipoles. The scaling is based on detailed measurements of few superconducting accelerator magnets belonging to two families, the LHC and Tevatron dipoles, and could have direct implications for accelerator control. In addition, we have given an interpretation of the scaling found, based on the behavior of the magnetization of a strand pre-magnetized in a rotating field and subjected to a subsequent field sweep. The analytical expression derived for the scaling can be used to complement measurements, as well as to predict the snapback for different magnet designs.

\section{REFERENCES}

[1] D. A. Finley et al., "Time dependent chromaticity changes in the tevatron," in 1987 Proc. Part. Acc. Conf., pp. 151-153.

[2] P. Bauer et al., "Analysis of the b2 correction in the tevatron," in 2003 Proc. Part. Acc. Conf., pp. 1730-1732.

[3] H. Brueck et al., "Correction of the influence of persistent currents in the HERA proton ring," in 1996 Proc. European Part. Acc. Conf., pp. 392-394.

[4] R. Stiening, "A possible mechanism for enhanced persistent current sextupole decay in SSC dipoles," SSCL-359, Jan. 1991.

[5] R. Wolf, "The decay of the field integral in superconducting accelerator magnets wound with Rutherford cables," in 1998 Proc. 15th Int. Magnet Tech. Conf., pp. 238-241.

[6] M. Haverkamp et al., "Interaction between current imbalance and magnetization in LHC cables," IEEE Trans. Appl. Supercond., vol. 11, pp. 1609-1612, 2001.

[7] M. Haverkamp, "Decay and Snapback in Superconducting Accelerator Magnets," Ph.D. Thesis, Twente University Press, 2003.

[8] L. Rossi, "The LHC superconducting magnets," in 2003 Proc. Part. Acc. Conf., pp. 141-145.

[9] H. D. Edwards, "Tevatron energy doubler: a superconducting accelerator," Ann. Rev. Nucl. Part. Sci., vol. 35, p. 605, 1985.

[10] L. Bottura et al., "A fast sextupole probe for snapback measurement in the LHC dipoles," IEEE Trans. Appl. Supercond., vol. 10, pp. 1435-1438, 2000.

[11] M. Haverkamp et al., "Field decay and snap-back measurements using a fast hall plate detector," IEEE Trans. Appl. Supercond., vol. 12, pp. 86-89, 2002.

[12] T. Pieloni et al., "Field decay and snapback measurements using a fast hall probe sensor," IEEE Trans. Appl. Supercond, vol. 14, pp. 1822-1825, 2004.

[13] L. Bottura et al., "A scaling law for predicting snap-back in superconducting accelerator magnets," in 2004 Proc. European Part. Acc. Conf., pp. 1609-1611.

[14] K.-H. Mess, P. Schmüser, and S. Wolff, Superconducting Accelerator Magnets: World Scientific, 1996. 